\title{
LAGRANGIAN TORI IN THE PROJECTIVE PLANE
}

\author{
N. A. Tyurin*
}

We extend the discussion of the homological mirror symmetry for toric manifolds to the more general case of monotonic symplectic manifolds with real polarizations. We claim that the Hori-Vafa conjecture, proved for toric Fano varieties, can be verified in a much wider context. Then the Bohr-Sommerfeld notion regarding the canonical class Lagrangian submanifold appears and plays an important role. A bridge is thus manifested between the geometric quantization and homological mirror symmetry programs for the projective plane in terms of its Lagrangian geometry. This allows using standard facts from the theory of geometric quantization to obtain some results in the framework of the theory of homological mirror symmetry.

Keywords: Lagrangian torus, projective plane, Bohr-Sommerfeld condition

\section{Introduction}

The Lagrangian geometry of compact symplectic manifolds remains a subject about which not much is known. Even in the simplest case of two-dimensional compact symplectic manifolds (Riemann surfaces), where the Lagrangian condition degenerates because any one-dimensional submanifold is Lagrangian, the classification problems (up to Hamiltonian isotopy or up to symplectomorphisms) are solved only for certain special cases including the case of the projective line. In four dimensions, it is unknown which two-dimensional manifolds appear as Lagrangian submanifolds, and the discussion of the existence of a Lagrangian Klein bottle in Kahler surfaces is still unfinished.

It is believed that the projective plane $\mathbb{C P}^{2}$ admits only Lagrangian tori as orientable Lagrangian submanifolds and real projective planes as nonorientable ones, plus some artificial types produced by hand using Lagrangian surgery near the intersections of Lagrangian tori and real projective planes, which gives new topological types of Lagrangian submanifolds such as $T^{2} \sharp \mathbb{R}^{2}$.

On the other hand, the classification of Lagrangian tori is incomplete even for $\mathbb{C P}^{2}$ : two types of Lagrangian tori are known (the Clifford and Chekanov types), and it seems that these types belong to different classes in the classification up to Hamiltonian isotopy (a paper is in preparation by Chekanov and Schlenk but is not yet available). Of course, this does not mean that the set of equivalence classes is exhausted by these Clifford and Chekanov types.

At the same time, Lagrangian geometry is highly desired for certain approaches to the mirror symmetry conjecture. According to the homological mirror symmetry program proposed by Kontsevich [1], the symmetry is an equivalence between the derived category of coherent sheaves over a given algebraic mani-

*Joint Institute for Nuclear Research, Dubna, Moscow Oblast, Russia; Moscow State University of Railway Engineering, Moscow, Russia, e-mail: ntyurin@theor.jinr.ru.

Prepared from an English manuscript submitted by the authors; for the Russian version, see Teoreticheskaya $i$ Matematicheskaya Fizika, Vol. 158, No. 1, pp. 3-22, January, 2009. Original article submitted March 4, 2008. 
fold $M$ and a Fukaya-Floer category of Lagrangian submanifolds of its mirror partner $\operatorname{mir}(M)$, which is a symplectic manifold. The objects of the Fukaya-Floer category are Lagrangian submanifolds up to Hamiltonian isotopy, and the morphisms are induced by the Floer cohomology of pairs of objects. Although a rigorous definition of the Floer cohomology does not exist in general, it is clear that the full category of Lagrangian submanifolds up to Hamiltonian isotopy is in any case too large, and this implies restrictions on the type of Lagrangian submanifolds taken in the specific constructions. In the four-dimensional case, a variant of the general Fukaya-Floer theory is adopted using a certain additional structure, the Lefschetz pencil on a given symplectic manifold. The Fukaya-Seidel category thus obtained is compared with the derived category of coherent sheaves; in the Fukaya-Seidel category, not all Lagrangian submanifolds but only vanishing cycles are taken.

Another type of restriction is proposed for toric Fano varieties: we take a toric fibration for such an $X$ and consider the fibers $S_{\alpha}$ (which are Lagrangian submanifolds in $X$ ) with a nontrivial Floer cohomology $\mathrm{FH}^{*}\left(S_{\alpha}, S_{\alpha} ; \mathbb{C}\right) \neq 0$. The needed category is then constructed over the set of the fibers satisfying this nontriviality condition. In this case, the Hori-Vafa conjecture states that the number of such fibers is finite and should be the Euler characteristic of the mirror partner [2]. This conjecture was proved in [3] under certain assumptions. The Bott-Morse version is computed instead of the Floer cohomology. The complex structure of the toric variety or its small Hamiltonian deformations is taken instead of a general almost complex structure. The answer looks very familiar in the framework of the geometric quantization of toric varieties: the desired fibers are distinguished by a certain integrality condition (see formula (10.6) in [3]), and this integrality condition in the toric framework means that we deal with Bohr-Sommerfeld fibers.

But the main idea of mirror symmetry is to relate the algebraic geometry of a given variety to the symplectic geometry of its mirror partner, and the answer on the right-hand side must therefore be independent of the choice of the compatible complex structure. A certain sufficiently general almost complex structure is used to construct the objects like the Gromov invariants or the Floer cohomology, and we know that an integrable complex structure is far from being general in this setup (many examples of answers that must be corrected are known in gauge theories, etc.). Moreover, the complex structure of a toric variety is even more special (any toric variety is rigid in the class of toric varieties but can, of course, be deformed to a nontoric algebraic variety). Therefore, we must extend the setup in [3] such that it would be more independent of the choice of the complex structure. On the other hand, doing this, we can see that the results in [3] can be understood as more general facts adopted to the specific case of the toric Fano varieties.

What is proposed as such a generalized consideration? We consider monotonic simply connected compact symplectic manifolds instead of Fano varieties. We consider symplectic manifolds with real polarizations instead of toric varieties. After such a generalization, we have a situation well known in geometric quantization (see, e.g., [4]-[6]).

Geometric quantization is a set of recipes assigning a given symplectic manifold appropriate Hilbert spaces together with homomorphisms of the Lie algebra of smooth functions on that manifold with values in the spaces of self-adjoint operators acting on these Hilbert spaces (or, more generally, it assigns a given symplectic manifold a certain algebraic variety [7]). One of the recipes can be used in the case where our given symplectic manifold admits an additional structure, a real polarization, which is a Lagrangian fibration of our given symplectic manifold. In this case, the Hilbert spaces are spanned by the fibers satisfying a specific condition, the so-called Bohr-Sommerfeld condition of a certain level. The crucial fact here is that the number of Bohr-Sommerfeld fibers in the compact case is finite if the Lagrangian fibration is "sufficiently good."

The toric Fano case is included in this class of "sufficiently good" Lagrangian fibration, and the construction in [3] ensures that the Bohr-Sommerfeld fibers then coincide with the fibers with a nontrivial adopted Floer-Bott-Morse cohomology. To avoid the ambiguity arising from different versions of the Floer 
cohomology, we universalize the situation, having in mind the following general fact: if a Lagrangian submanifold is displaceable, then it should have a trivial Floer cohomology for any version of the Floer cohomology theory; if a Lagrangian submanifold is monotonic, then it should have a nontrivial cohomology for any definition. We recall that $S$ is displaceable if it can be moved by some Hamiltonian isotopy $\psi_{\mathrm{H}}$ such that the intersection $S \cap \psi_{\mathrm{H}}(S)=\varnothing$. Of course, the presented distribution, generally speaking, is not complete, but at least for the basic example of a monotonic simply connected symplectic manifold with real polarization (the projective plane), all currently known examples agree with such an implication.

This paper contains results about the fibers of a real polarization with regular degeneration. The number of monotonic fibers for a general monotonic symplectic manifold is finite (Theorem 2). For any real polarization of the projective plane with regular degeneration, any Bohr-Sommerfeld fiber with respect to the canonical class is monotonic, and any other fiber is displaceable (Theorem 3). We emphasize that this holds for any real polarization with regular degeneration, not just for a toric one. We do not use the toric structure in the constructions below and in parallel present several (naive) conjectures extending the statement in Theorem 3 to the case of any Lagrangian torus in $\mathbb{C P}^{2}$, not just for the fibers. It is currently difficult to expect that all these conjectures are true, but we continue to work in this direction.

The discussion below follows the idea that the Bohr-Sommerfeld condition and the nontriviality condition for the Floer cohomology are somehow related. If this is indeed the case, then we would obtain a way to proceed with the homological mirror symmetry using known results and constructions in geometric quantization. Our main motivation for studying this question is that it would realize the ideology proposed by A. N. Tyurin that claims that mirror symmetry and geometric quantization are related [8].

\section{Bohr-Sommerfeld conditions}

We consider a compact simply connected symplectic manifold $(M, \omega)$ of real dimension $2 n$ and assume that the cohomology class $[\omega] \in H^{2}(M, \mathbb{R})$ is integer. This means that there exists a complex line bundle $L \rightarrow M$ with the first Chern class $c_{1}(L)=[\omega]$. Choosing a Hermitian structure on $L$, we obtain the space of Hermitian connections $\mathcal{A}_{h}(L)$. There exists a Hermitian connection $a \in \mathcal{A}_{h}(L)$ unique up to gauge transformations such that its curvature form $F_{a}$ is proportional to $\omega$ :

$$
F_{a}=2 \pi i \omega
$$

The pair $(L, a)$ is usually called the prequantization data. We consider an arbitrary integer $k \in \mathbb{Z}$ and the corresponding power $L^{k}$. The space $\mathcal{A}_{h}\left(L^{k}\right)$ contains a Hermitian connection $a_{k}$ unique up to gauge transformations such that its curvature form is proportional to $\omega$ :

$$
F_{a_{k}}=2 k \pi i \omega
$$

The pair $\left(L^{k}, a_{k}\right)$ is called the prequantization data of level $k$.

Let $S \subset M$ be a Lagrangian submanifold. This means that $S$ has the dimension $n$ and the restriction $\left.\omega\right|_{S}$ vanishes identically. Restricting the pair $\left(L^{k}, a_{k}\right)$ to $S$, we then obtain a trivial line bundle with a flat connection (the curvature form vanishes because it is proportional to the symplectic form). Therefore, Lagrangian submanifolds can be identified in terms of the flat connections thus obtained.

We say that a Lagrangian submanifold $S \subset M$ is a Bohr-Sommerfeld manifold of level $k$ if the restricted connection $\left.a_{k}\right|_{S}$ admits covariantly constant sections.

It could be asked whether this definition depends on the choices of the Hermitian structure on $L$ and a connection $a$ from the equivalence class of Hermitian connections described by the condition $F_{a}=2 \pi i \omega$. The point is that the definition is absolutely universal: it can be reformulated in our case as follows. We consider $H_{1}(S, \mathbb{Z})$ and some representative $\gamma_{b} \subset S$ for an arbitrary primitive element $b \in H_{1}(S, \mathbb{Z})$. Because 
$\pi_{1}(M)$ is trivial, we can find a $\operatorname{disc} D \subset M$ with the boundary $\partial D=\gamma_{b}$. It is easy to see that $S$ is a Bohr-Sommerfeld manifold of level $k$ if and only if the symplectic area of $D$ times $k$ is integer for any $b \in H_{1}(S, \mathbb{Z})$ :

$$
k \int_{D} \omega \in \mathbb{Z}
$$

We note that we consider the case where $[\omega] \in H^{2}(M, \mathbb{Z}) \subset H^{2}(M, \mathbb{R})$, and the last integrality condition is independent of the choice of $D$. At the same time, it does not involve any bundles or connections, and the notion of the Bohr-Sommerfeld condition is therefore universal. It is natural to call the numbers

$$
p_{k}(b)=k \int_{D} \omega \quad(\bmod \mathbb{Z})
$$

the periods of the given Lagrangian submanifold.

On the other hand, the last description relates local deformations of a given symplectic manifold to variations of the periods of the deformed submanifolds. According to the Darboux-Weinstein theorem [9], there exists a certain small tubular neighborhood of the given $S$ symplectomorphic to a neighborhood of the zero section in $T^{*} S$ endowed with the standard symplectic form. Local Lagrangian deformations of $S$ are then represented by graphs of closed 1-forms on $S$. With the period description in mind, we can see that a deformation $\psi$ of a given $S$ with the class $[\psi] \in H^{1}(S, \mathbb{R})$ changes the periods as

$$
p_{k}\left(\psi_{*}(b)\right)=p_{k}(b)+k \psi(b) .
$$

Indeed, if we have a loop $\gamma_{b}$ on the given Lagrangian submanifold and deform it to $\psi\left(\gamma_{b}\right)$, then the symplectic area of the tube with the boundary $\gamma_{b}-\psi\left(\gamma_{b}\right)$ is exactly $\psi(b)$, which implies formula (1).

As a corollary, we obtain the existence of a Bohr-Sommerfeld Lagrangian submanifolds of (perhaps, sufficiently large) level $k$ if there exists an arbitrary Lagrangian submanifold.

A variant of the basic definition appears in the case of a monotonic symplectic manifold. A symplectic manifold $(M, \omega)$ is said to be monotonic if its canonical class $K_{\omega} \in H^{2}(M, \mathbb{Z})$ is proportional to $[\omega]$ :

$$
K_{\omega}=k[\omega]
$$

The number $k$ is called the monotonicity coefficient. For any Hermitian structure on $K_{\omega}$ in this case, there exists a Hermitian connection $a_{\text {can }}$ unique up to gauge transformations with its curvature form proportional to the symplectic form. We repeat the basic definition with respect to $\left(K_{\omega}, a_{\mathrm{can}}\right)$. If the restriction $\left.\left(\omega, a_{\text {can }}\right)\right|_{S}$ admits covariantly constant sections, then we say that $S$ is a Bohr-Sommerfeld manifold with respect to the canonical class. Such a specification is justified; we discuss it in Sec. 3.

\section{Finiteness}

We assume that we have an additional structure on $M$, a real polarization. This means that $M$ is fibered over a base $B$ and almost all the fibers are smooth Lagrangian fibers. This usually happens for phase spaces of completely integrable systems, and there hence exists a commutative subalgebra in the Poisson algebra $\left(C^{\infty}(M, \mathbb{R}),\{\cdot, \cdot\}_{\omega}\right)$ spanned by the set of smooth functions $\left\{f_{1}, \ldots, f_{n}\right\}$, called integrals, such that the differentials $\left(d f_{1}, \ldots, d f_{n}\right)$ form a basis in the cotangent space almost everywhere on $M$. These conditions dictate several topological restrictions; for us, the most important is that a smooth fiber must be isomorphic to a torus.

For a real polarization of $M$ given by a map

$$
\pi: M \rightarrow B
$$


where $B$ is a convex polytope in $\mathbb{R}^{n}$, we have the so-called Kodaira-Spencer map. For each regular point $b \in B$, the deformation along the base somehow corresponds to the deformation of the fiber $\pi^{-1}(b) \subset M$, and because the local deformations of a Lagrangian submanifold are described by closed 1-forms, we have the map

$$
m_{\mathrm{KS}}: T_{b} B \rightarrow H^{1}\left(\pi^{-1}(b), \mathbb{R}\right) .
$$

We claim that for a smooth fiber $\pi^{-1}(b)$, the Kodaira-Spencer map is an isomorphism. To prove this, we first note that the dimensions of the compared spaces are the same:

$$
\operatorname{dim} T_{b} B=n=\operatorname{dim} H^{1}\left(T^{n}, \mathbb{R}\right) .
$$

If we suppose that a vector $v \in T_{b} B$ goes to zero under this map, then this would imply that the corresponding small deformation is isodrastic, i.e., it preserves the periods of $S_{b}=\pi^{-1}(b)$. But according to formula (1), this could happen if and only if the corresponding closed 1-form $\psi$ is exact. In other words, there exists a smooth function $f$ on $S_{b}$ such that $\psi=d f$. But each smooth function must have at least two critical points on a compact manifold, a maximum and minimum. This means that the graph of $\psi$ in this case must intersect our given $S_{b}$ in at least two points. But this is impossible because the fibers do not intersect each other. Therefore, the Kodaira-Spencer map does not have a kernel and by the dimensional argument is an isomorphism.

In geometric quantization, the approach with a real polarization gives the following recipe for constructing the Hilbert spaces [5]. In a fixed real polarization, we take the fibers $S_{1}, \ldots, S_{l}, \ldots$ that are Bohr-Sommerfeld of level $k$ and then consider the linear span

$$
\sum_{i} \mathbb{C}\left\langle S_{i}\right\rangle=\mathcal{H}_{k} .
$$

The point is that the set of such fibers is discrete in any case and finite if the real polarization has sufficiently good degenerations. Indeed, the discreteness follows simply because the Kodaira-Spencer map is an isomorphism. Just what are the "sufficiently good degenerations"? They appear in the case of toric varieties, for example. This means that the degenerations are regular, i.e., if $B$ is a convex polytope in $\mathbb{R}^{n}$, then the fibers over the inner part are smooth. The picture over an arbitrary $(n-1)$-dimensional face is repeated, i.e., a smooth symplectic manifold fibered over this face with smooth Lagrangian fibers over the inner part of this face (which are smooth Lagrangian $(n-1)$-dimensional tori), and so on. In this situation, the number of smooth Bohr-Sommerfeld Lagrangian fibers is finite.

Indeed, the discrete set can have a limit point only on the boundary. We suppose that the limit point corresponds to a smooth $(n-1)$-dimensional torus placed over the inner part of a $(n-1)$-dimensional face. The preimage of this $(n-1)$-dimensional face is a symplectic submanifold $M_{1} \subset M$. The limit process implies that for our fixed $k$ (the level by the Bohr-Sommerfeld property), the normal bundle $\left.N_{M_{1} / M}\right|_{S_{\text {lim }}}$, where $S_{\lim }$ is a degenerate torus corresponding to the given limit point, contains a series of shrinking disc bundles each of which consists of discs of constant symplectic area such that this area times $k$ is an integer. This implies that starting with some sufficiently small disc bundle, the symplectic area of the fiber discs must be zero. But this is impossible because the normal bundle $N_{M_{1} / M}$ is symplectic and each disc must have a nontrivial symplectic volume. Therefore, the limit point cannot exist on the inner part of a $(n-1)$-dimensional face.

The same arguments with lower dimensions show that the limit point can appear only if it corresponds to a zero-dimensional edge of $B$ and hence to a vertex $p \in B$ of a polytope. Choosing some Darboux coordinates in a neighborhood of $\pi^{-1}(p) \in M$ and representing the sequence of shrinking tori in these coordinates starting with a certain number, we obtain a contradiction.

We thus obtain the following statement. 
Theorem 1. Let $X$ be a simply connected symplectic manifold and $\pi: X \rightarrow B$ be a real polarization with regular degenerations. Then for any level $k \in \mathbb{Z}$, the set of Bohr-Sommerfeld Lagrangian fibers of level $k$ is finite.

\section{Monotonicity}

As already mentioned in Sec. 1, there is a variant of the Bohr-Sommerfeld condition in the case of monotonic symplectic manifolds. In the Floer cohomology theory, one of the most important cases is where a given Lagrangian submanifold is monotonic. To describe such a manifold, we first recall what the Maslov index is. Because we discuss Lagrangian tori below, we restrict ourself to the case of orientable Lagrangian submanifolds.

Let $S \subset M$ be an orientable Lagrangian submanifold of a simply connected symplectic manifold $(M, \omega)$. We choose an arbitrary almost complex structure compatible with $\omega$ and realize the anticanonical bundle $K_{\omega}^{-1}$ using the determinant of the Hermitian bundle $(T M, I, \omega)$. For an arbitrary loop $\gamma \subset S$, we choose a disc $D \subset M$ with the boundary $\partial D=\gamma$ and consider a trivialization of the anticanonical bundle $\left.K_{\omega}^{-1}\right|_{D}$ restricted to $D$. This trivialization is unique up to gauge transformations, and because $D$ is simply connected, the degree of all such transformations computed on the boundary must be trivial. In this realization, the chosen trivialization is represented by a type- $(n, 0)$ polyvector field $\eta$ that is nonvanishing on $D$. On the other hand, the disc boundary carries a nonvanishing real polyvector field $\theta$ given by the determinant of $T S$ restricted to $\gamma$. Therefore, the Hermitian pairing of $\eta$ and $\theta$ gives a map

$$
\phi_{D}: \gamma \rightarrow \mathbb{C}^{*}
$$

because it is easily seen that the Lagrangian condition implies that $\langle\eta, \theta\rangle_{h}$ never vanishes. The degree of this map

$$
\mu(\gamma, D)=\left\langle\phi_{D}^{*} h ;[\gamma]\right\rangle
$$

(where $h$ is the generator of $H^{1}\left(\mathbb{C}^{*}, \mathbb{Z}\right)$ and $[\gamma] \in H_{1}(\gamma, \mathbb{Z})$ is the fundamental class) is an integer that is independent of the choice of the almost complex structure. Moreover, it is independent of the choice of $D$ in the same class from $\pi_{2}(M, S)$ with the image at $[\gamma] \in \pi_{1}(S)$ under the canonical homomorphism. For another disc with the same boundary $\gamma$, the value $\mu\left(\gamma, D^{\prime}\right)$ can be computed as

$$
\mu\left(\gamma, D^{\prime}\right)=\mu(\gamma, D)+\left\langle K_{\omega}^{-1} ;\left[S^{2}=D \cup D^{\prime}\right]\right\rangle
$$

Hence, if $D^{\prime}$ is homotopy equivalent to $D$, then the numbers must be the same. At the same time, the number is independent of the choice of $\gamma$ in a given class $[\gamma] \in \pi_{1}(S)$. In summary, we have a map

$$
\mu: \pi_{2}(M, S) \rightarrow \mathbb{Z}
$$

which is called the Maslov index. For any simply connected symplectic manifold and any Lagrangian submanifold, this index exists and, moreover, is invariant under any Lagrangian deformation. As follows from its definition, it must be invariant under any continuous deformations. In the case where the ambient symplectic manifold has a small second cohomology $(\operatorname{Pic} M=\mathbb{Z})$, the index can be reduced to the numerical correspondence

$$
\mu: H_{1}(S, \mathbb{Z}) \rightarrow \mathbb{Z} \quad\left(\bmod \operatorname{deg} K_{\omega}^{-1}\right),
$$

which is often called the Maslov number. 
Further, a Lagrangian submanifold $S \subset M$ is monotonic if there exists an integer $k$ such that for any loop $\gamma \subset S$ and any disc $D \subset M, \partial D=\gamma$, we have

$$
\mu(\gamma, D)=k \int_{D} \omega
$$

where $\mu$ is the Maslov index of the loop $\gamma$ with respect to the disc $D$. The existence of a monotonic Lagrangian submanifold imposes strong restrictions on the topology of $M$ : it must itself be monotonic. Consequently, it is natural to use the notion of Bohr-Sommerfeld Lagrangian submanifolds with respect to the canonical bundle. It is easy to see that a Lagrangian submanifold is monotonic only if it is a Bohr-Sommerfeld manifold with respect to the canonical class. Indeed, identity (2) is possible only in the case where the symplectic area of $D$ times $k$ is an integer for arbitrary $\gamma$ and $D$. But this is exactly our Bohr-Sommerfeld condition with respect to the canonical class.

On the other hand, for a Bohr-Sommerfeld Lagrangian submanifold with respect to the canonical bundle in a monotonic simply connected symplectic manifold, we can define a characteristic class called the universal Maslov class [10]. Setting its first definition aside, we here define it as follows. For a given Bohr-Sommerfeld submanifold $S \subset M$ with respect to the canonical class with $K_{\omega}^{-1}=k[\omega]$ for an arbitrary loop $\gamma$ and disc $D, \partial D=\gamma$, we consider the difference

$$
m_{S}(\gamma, D)=\mu(\gamma, D)-k \int_{D} \omega \in \mathbb{Z}
$$

Then the value of $m_{S}$ is independent of the choice of $D$. Moreover, this numerical correspondence is linear, and $m_{S}$ is consequently a cohomology class from $H^{1}(S, \mathbb{Z})$. We recall that this class is well defined if and only if our Lagrangian submanifold is a Bohr-Sommerfeld manifold with respect to the canonical bundle. And because this property is stable only under Hamiltonian deformations, the resulting cohomology class is invariant under Hamiltonian deformations [10].

It follows tautologically from this description that $S$ is monotonic if and only if it is a Bohr-Sommerfeld manifold with respect to the canonical class and its universal Maslov class vanishes, $m_{S}=0$. Summarizing our discussion, we have the following statement, whose proof is obvious.

Theorem 2. Let $X$ be a simply connected monotonic symplectic manifold and $\pi: X \rightarrow B$ be a real polarization with regular degenerations. Then the number of monotonic Lagrangian fibers is finite.

\section{Lagrangian tori in $\mathbb{C P}^{2}$}

In what follows, we discuss the basic example of a monotonic simply connected symplectic manifold, the projective plane $\mathbb{C P}^{2}$. We take the projective plane $\mathbb{C P}^{2}$ with the standard Fubini-Study Kahler form $\omega$, which we regard as a symplectic form. Hence, the cohomology class $[\omega]$ is integer and represents a generator of $H^{2}\left(\mathbb{C P}^{2}, \mathbb{Z}\right)$. As a symplectic manifold, the projective plane is monotonic, $K=-3[\omega]$. We are interested in Lagrangian fibrations of $\mathbb{C P}^{2}$. We use them to verify the following naive conjecture.

Conjecture 1. If a smooth Lagrangian fiber is displaceable, then it is not Bohr-Sommerfeld with respect to the canonical class, and if this fiber is Bohr-Sommerfeld with respect to the canonical class, then it is also monotonic.

This conjecture can be rewritten for an arbitrary simply connected monotonic symplectic manifold endowed with a real polarization with regular degenerations. Below, we show that this conjecture holds for the projective plane, but we first present the basic example of Lagrangian tori in $\mathbb{C P}^{2}$. 
The first and simplest example of Lagrangian fibration of $\mathbb{C P}^{2}$ comes from toric geometry. We choose homogeneous coordinates $\left[z_{0}: z_{1}: z_{2}\right]$ and consider the subset of $\mathbb{C P}^{2}$ defined by the system of equations

$$
z_{j}=r_{j} e^{i \phi_{j}}, \quad j=0,1,2,
$$

where $r_{j}$ are fixed positive real numbers satisfying $r_{0}+r_{1}+r_{2}=1$ and $\phi_{j}$ are real parameters. In $\mathbb{C}^{3}$, this would give a three-dimensional torus, but after factoring by phase rotations, we obtain a smooth two-dimensional torus in $\mathbb{C P}^{2}$. Varying the $r_{j}$, we obtain a family of Lagrangian tori, i.e., a Lagrangian fibration of $\mathbb{C P}^{2}$ over a triangle $\Delta \subset \mathbb{R}^{2}$. Indeed, we can assign each smooth torus a pair $\left(r_{0}, r_{1}\right)\left(r_{2}\right.$ is uniquely defined by $r_{0}$ and $r_{1}$ ); all possible values of $r_{0}$ and $r_{1}$ form the triangle $\Delta$. The degenerations of this Lagrangian fibration are regular: over the segments $\left\{r_{0}=0,0<r_{1}<1\right\},\left\{r_{1}=0,0<r_{0}<1\right\}$, and $\left\{r_{0}+r_{1}=1,0<r_{1,2}<1\right\}$, we have one-dimensional toric fibers, and the vertices of $\Delta$ correspond to the maximum degenerations, zero-dimensional tori or simply points. We let $T_{r_{0}, r_{1}}$ denote the smooth fiber of $\left(r_{0}, r_{1}\right) \in \Delta$. Such tori are called Clifford tori, and the fibration is called the Clifford fibration of the projective plane.

Because the symplectic form is integer, the question arises about the Bohr-Sommerfeld fibers of this fibration. The line bundle $L=\mathcal{O}(1)$ with a Hermitian connection $a$ whose curvature form is proportional to the symplectic form distinguishes a set of Bohr-Sommerfeld fibers of different levels. And the specification is very simple: the fiber $T_{r_{0}, r_{1}}$ is Bohr-Sommerfeld of level $k$ if and only if

$$
k r_{0}, k r_{1} \in \mathbb{Z}
$$

Indeed, the periods of the torus $T_{r_{0}, r_{1}}$ are given by the numbers $r_{0}$ and $r_{1}$ with respect to the appropriate generators of $H_{1}\left(T_{r_{0}, r_{1}}, \mathbb{Z}\right)$, which implies the statement. It is hence obvious that

1. there are no Bohr-Sommerfeld fibers of levels 1 and 2,

2. there is a unique fiber that is Bohr-Sommerfeld of level 3 and is therefore Bohr-Sommerfeld with respect to the canonical class, and

3. the number of fibers that are Bohr-Sommerfeld of level $k$ is exactly $\operatorname{dim} H^{0}\left(\mathbb{C P}^{3}, \mathcal{O}(k-3)\right)$.

The last coincidence can be promoted to the direct equality "the number of $k$-Bohr-Sommerfeld fibers is equal to the dimension of the holomorphic section space of $\mathcal{O}(k)$ " if we generalize the situation and consider singular fibers together with the smooth ones. Then there would be exactly three Bohr-Sommerfeld fibers of level 1 (the three points over the vertices of $\Delta$, zero-dimensional tori), six Bohr-Sommerfeld fibers of level 2 (the same three points plus three equatorial one-dimensional tori over the triangle edges), ten BohrSommerfeld fibers of level 3 (the same three points plus two one-dimensional tori on the edges of the triangle $\Delta$ plus our regular fibers), and so on. This effect is known in the geometric quantization of toric varieties.

We are interested in only regular fibers here, and we therefore consider what would be the result of applying the approach described above in the homological mirror symmetry. To start, we take the fibers with a nontrivial Floer cohomology. Setting possible definitions of the Floer cohomology $\mathrm{FH}^{*}\left(S, S ; \mathbb{Z}_{2}\right)$ aside, we can here use our interpretation: if a Lagrangian submanifold $S$ is displaceable, then it has a trivial Floer cohomology. Displaceability means that there exists a Hamiltonian isotopy $\psi_{t}$ such that $\psi_{t}(S)$ does not intersect $S$ for some $t$ :

$$
\psi_{t}(S) \cap S=\varnothing .
$$

It is easy to see that if $r_{0}$ and $r_{1}$ are both not equal to $1 / 3$, then $T_{r_{0}, r_{1}}$ is displaceable. Indeed, for $\mathbb{C P}^{2}$, we have the subalgebra of symbols in the Poisson algebra $\left(C^{\infty}\left(\mathbb{C P}^{2}\right),\{\cdot, \cdot\}_{\omega}\right)[7]$ corresponding to 
self-adjoint operators on $\mathbb{C}^{3}$. The Hamiltonian flow sending $T_{r_{0}, r_{1}}$ to $T_{r_{1}, r_{0}}$ is induced by the self-adjoint operator interchanging $z_{0}$ and $z_{1}$ in $\mathbb{C}^{3}$. Because the fibers do not intersect each other, we find that if $r_{0} \neq r_{1}$, then $T_{r_{0}, r_{1}}$ is displaceable, and the same holds if $r_{1} \neq 1-r_{0}-r_{1}$. One absolutely symmetric possibility remains, when $r_{i}=1 / 3, i=0,1,2$, but this is precisely the Bohr-Sommerfeld Lagrangian fiber with respect to the canonical class. To examine whether it has a nontrivial Floer cohomology, we use the following argument: the given Lagrangian torus $T_{1 / 3,1 / 3}$ is monotonic. Indeed, it is Bohr-Sommerfeld with respect to the canonical class and is minimal; therefore, the universal Maslov class is trivial [10]. This fact was used in [11] to prove that the Floer cohomology of $T_{1 / 3,1 / 3}$ is isomorphic to its de Rham cohomology,

$$
\mathrm{FH}^{*}(S, S ; \mathbb{C})=H_{\mathrm{dR}}^{*}(S, \mathbb{C}),
$$

which is, of course, well known for a torus.

Hence, for the standard toric fibration of $\mathbb{C P}^{2}$ (and the same holds for any projective space), the condition of being Bohr-Sommerfeld with respect to the canonical class is equivalent to the monotonicity condition and, furthermore, to the nondisplaceability condition (in particular, the results in [11] follow from it). There is a simple natural extension of the toric case: a Lagrangian torus in $\mathbb{C P}^{2}$ is said to be of the Clifford type if there exists a Hamiltonian isotopy sending this torus to a standard fiber of the Clifford fibration. The Floer cohomology is invariant under Hamiltonian deformations (this is its main property, which could even be taken as its general definition). Moreover, the Bohr-Sommerfeld condition of an arbitrary level, the monotonicity condition, and the nondisplaceability condition are also preserved under a Hamiltonian deformation. Therefore, what was said above also holds for any torus of the Clifford type.

We complete our discussion of tori in $\mathbb{C P}^{2}$ of the Clifford type, noting in summary that Conjecture 1 holds for the standard toric fibration of $\mathbb{C P}^{2}$. The extension of Conjecture 1 to the case of the projective plane can be formulated as a series of conjectures, which are rather naive and are currently supported only by verification in known examples. All these conjectures become theorems if Lagrangian tori are replaced with fibers of a real polarization with regular degenerations. We show this below, but we formulate the conjectures in general form here.

Conjecture 2. If $S \subset \mathbb{C P}^{2}$ is a Bohr-Sommerfeld Lagrangian torus of level $k$, then necessarily $k \geq 3$.

If this conjecture holds, then the class of Bohr-Sommerfeld Lagrangian tori with respect to the canonical class is "primary" in the sense that any Bohr-Sommerfeld Lagrangian torus of level 1 should a priori be included in the set of Bohr-Sommerfeld Lagrangian tori with respect to the canonical class (because 3 is divisible by 1), but such a torus strongly differs symplectically from "pure" Bohr-Sommerfeld tori with respect to the canonical class.

Conjecture 3. The universal Maslov class of any Bohr-Sommerfeld Lagrangian torus $S \subset \mathbb{C P}^{2}$ with respect to the canonical class is trivial,

$$
H^{1}(S, \mathbb{Z}) \ni m_{S}=0
$$

If this conjecture holds, then every Bohr-Sommerfeld Lagrangian torus with respect to the canonical class must be monotonic and must therefore have a nontrivial Floer cohomology. This implies our third suggestion.

Conjecture 4. A smooth Lagrangian torus $S \subset \mathbb{C P}^{2}$ of the projective plane is nondisplaceable if and only if $S$ is a Bohr-Sommerfeld torus with respect to the canonical class. 
We note that all these conjectures are stronger than needed for proving a general version of the HoriVafa conjecture: it suffice to use a weaker statement.

Conjecture 5. Let $X$ be a monotonic simply connected symplectic manifold and $\pi: X \rightarrow B$ be a real polarization. Then a smooth fiber $\pi^{-1}(b)=S_{b}$ is nondisplaceable if it is Bohr-Sommerfeld with respect to the canonical class.

We consider two nontoric examples.

Play example. We consider $\mathbb{C P}^{1}=S^{2}$ endowed with the standard symplectic form. Any smooth loop $\gamma \subset \mathbb{C P}^{1}$ is a Lagrangian submanifold, and the topological type of a smooth Lagrangian submanifold is actually exhausted by the one-dimensional torus $T^{1}$. The line bundle $L=\mathcal{O}(1)$ together with the appropriate Hermitian connection $a \in \mathcal{A}_{h}(L)$ defines the Bohr-Sommerfeld condition of level $k$, which in this case is as follows: a smooth loop $\gamma \subset \mathbb{C P}^{1}$ is Bohr-Sommerfeld of level $k$ if and only if it divides the surface into two pieces both of a symplectic area in $k^{-1} \cdot \mathbb{Z}$. This means that $\gamma \subset \mathbb{C P}^{1}$ is Bohr-Sommerfeld with respect to the canonical class if and only if it divides the surface into equal pieces. On the other hand, this is the case only if $\gamma$ is nondisplaceable. This means that Conjecture 5 holds for a smooth loop in $\mathbb{C P}^{1}$.

Of course, this says almost nothing about any other case: it follows because for a smooth loop in $\mathbb{C P}^{1}$, there is only one symplectic invariant that uniquely characterizes the loop up to a symplectomorphism, namely, the symplectic area of the disc bounded by this loop. But this is not true for higher dimensions: there is at least one more type of Lagrangian tori in $\mathbb{C P}^{2}$, called the Lagrangian tori of the Chekanov type [12], [13].

Nontoric example. This example was suggested in [13], where it was characterized as a nontoric fibration of $\mathbb{C P}^{2}$. We consider the family of conics $\left\{Q_{\varepsilon}\right\}$ in $\mathbb{C P}^{2}$ given by the equation

$$
Q_{\varepsilon}=\left\{z_{0} z_{1}=\varepsilon z_{2}\right\}
$$

where $\varepsilon \in \overline{\mathbb{C}}$ and $\left[z_{0}: z_{1}: z_{2}\right]$ are homogeneous coordinates. For this pencil with the basis points $[0: 1: 0]$ and $[1: 0: 0]$, we have exactly two singular conics: the conic is two intersecting lines for $\varepsilon=0$ and the conic degenerates into the double line $z_{2}^{2}=0$ for $\varepsilon=\infty$.

We consider the one-dimensional real subfamily in $\left\{Q_{\varepsilon}\right\}$ consisting of the conics $Q_{a e^{i t}-\mu}$, where $\mu \in \mathbb{C}^{*}$ and $a \in \mathbb{R}^{+}$are fixed numbers and $t$ is a real parameter. For each element in this subfamily, for example, $Q_{a \cdot e^{i t_{0}}-\mu}$, we have the natural fibration

$$
\pi: Q_{a e^{i t_{0}-\mu}} \rightarrow(-1,1) \subset \mathbb{R}
$$

given by the Hamiltonian action of the symbol preserving each conic in the family (this symbol is essentially unique up to a scaling [14]). The fiber can be labeled by $\delta \in(-1,1)$, and this correspondence has a certain meaning: the symplectic area of the disc bounding the loop $T_{t_{0}, \delta}^{1}$ equals $\delta(\bmod \mathbb{Z})$. Fixing a value of $\delta$, we distinguish the corresponding fiber $T_{t_{0}, \delta}^{1}$. We now vary $t_{0}$ in the real subfamily $\left\{Q_{a e^{i t}-\mu}\right\}$; this gives the corresponding family $T_{t, \delta}^{1}$, which forms a certain two-dimensional torus $T_{\delta}^{2}=T_{a, \mu, \delta}^{2} \subset \mathbb{C P}^{2}$. The point is that this torus is Lagrangian [13]. All tori thus constructed for different $a$ form a certain fibration of $\mathbb{C P}^{2} \backslash l_{2}$, where $l_{2}$ is the line $z_{2}=0$ with only one singular torus $T_{|\mu|, \mu, 0}^{2}$ with one shrunk loop (it is therefore not a real polarization with regular degenerations).

It was stated in [13] that for a fixed $\mu \neq 0$, the fibration of $\mathbb{C P}^{2}$ consists of two types of Lagrangian tori: the torus $T_{a, \delta}$ is of the Clifford type if $a>|\mu|$ and of the Chekanov type if $a<|\mu|$, and these types are different. 
What does this example yield for the discussion of our themes? We note the following:

1. The tori $T_{a, \delta}^{2}, a>|\mu|$, of the Clifford type were already discussed above, and Conjecture 5 holds for them.

2. Every torus $T_{a, \delta}^{2}, a<|\mu|$, of the Chekanov type is displaceable and therefore has a trivial Floer cohomology to itself.

3. There is no torus of the Chekanov type that is Bohr-Sommerfeld with respect to the canonical class.

Indeed, it is easy to construct a smooth function on $\mathbb{C P}^{2}$ whose Hamiltonian flow sends $T_{a, \mu, \delta}^{2}$ to $T_{a,-\mu, \delta}^{2}$. This function is far from general; it is a symbol corresponding to the self-adjoint operator $A=\operatorname{diag}(0,0,1)$. Its Hamiltonian flow acts as the rotation of the parameter space $\mathbb{C}$ with the two fixed points 0 and $\infty$. Because a torus of the Chekanov type corresponds to the case where two circles of the same radius $a$ with centers at $\mu$ and $-\mu$ do not intersect each other, we then find that such a torus is displaceable.

On the other hand, we show that a torus of the Chekanov type cannot be Bohr-Sommerfeld of level 1,2 , or 3 . We first take an arbitrary "equatorial" torus $T_{a, 0}$, where $a<|\mu|$ and $\delta=0$. For this torus, we have a distinguished basis in $H_{1}\left(T_{a, 0}, \mathbb{Z}\right)$ given by the loops $\alpha$ and $\beta$, where

$$
\alpha=T_{a, 0} \cap Q_{a}, \quad \beta \cup \beta^{\prime}=T_{a, 0} \cap l_{\left(z_{0}=z_{1}\right)},
$$

and $l$ is the projective line in $\mathbb{C P}^{2}$ given by the equation $z_{0}=z_{1}$. The intersection of $\alpha$ has two components of the same homological class, and each of them can be taken as the second element in our basis. The first loop $\alpha$ is the boundary of half of the conic $Q_{a}$ (is an equator), and the period of $\alpha$ is hence trivial (the symplectic area of each conic is equal to 2, and the area of half of a conic is hence equal to 1). This intersection is given by the equation

$$
z_{0}^{2}=\left(\mu-a e^{i \phi}\right) z_{2}^{2}
$$

on the projective line $l$, and the loops $\beta$ and $\beta^{\prime}$ are therefore given by the square roots of $\mu-a e^{i \phi}$. For any $\mu \in \mathbb{C}$ and $a \in \mathbb{R}, a<|\mu|$, the area of the subset

$$
z_{0}=\left(\mu-a e^{i \phi}\right)^{1 / 2} z_{2}
$$

is less than a quarter of the symplectic area of the entire projective line $l$. This means that the period of $\beta$ belongs to the interval $(0,1 / 4)$. On the other hand, if $T_{a, 0}$ were Bohr-Sommerfeld of level 1,2 , or 3 , then its periods would necessarily be integer after multiplication by 1,2 , or 3 . While the first period, corresponding to $\alpha$, is itself integer, the second period, corresponding to $\beta$, does not satisfy this condition.

To show that the same holds for any other $T_{a, \delta}$ with a nontrivial $\delta$, we use the monotonicity property of the periods for a smooth part of our Lagrangian fibration. We fix an arbitrary torus $T_{a_{0}, \delta_{0}}$ of the Chekanov type and consider a subset $M \subset \mathbb{C P}^{2}$ consisting of tori of the Chekanov type,

$$
M=\left\{T_{a, \delta} \mid a \leq a_{0}\right\}
$$

For this $M$, we have a real polarization with regular degenerations corresponding to the Lagrangian fibration of the "basis" conic $Q_{\mu}$. Hence, we can identify the fundamental groups of all tori in $M$ and use the basis of $\alpha$ and $\beta$ constructed above for the torus $T_{a, 0}$. As $a \rightarrow 0$, the basis element $\beta$ vanishes, and the Lagrangian fibration of $Q_{\mu}$ is given by only the element $\alpha$. It is therefore clear that such a real polarization has regular degenerations. We consider a lift of the period map to the two-component function

$$
f=\left(f_{1}, f_{2}\right): M \rightarrow \mathbb{R}^{2}
$$


such that

$$
\left.f_{i}\right|_{T_{a, \delta}}=\text { const },\left.\quad f_{i}\right|_{T} \equiv p_{i}(T) \quad(\bmod \mathbb{Z}) .
$$

Because $M$ is simply connected, such a lift exists. The obtained function $f=\left(f_{1}, f_{2}\right)$ is smooth on the inner part of $M$ and continuous on the entire $M$. It is monotonic, i.e., has no critical points, because the Kodaira-Spencer map is the differential of this function. Because the family of conics $Q_{\varepsilon}$ admits the symmetry $z_{0} \leftrightarrow z_{1}$, we can take the lift with the induced (skew) symmetry

$$
f_{1}\left(T_{a,-\delta}\right)=-f_{1}\left(T_{a, \delta}\right)
$$

It follows from the strict monotonicity condition that the image $f(M) \subset \mathbb{R}^{2}$ must be convex. It follows from the symmetry condition that it must be symmetric. We know that for the "equatorial" tori, $f_{2}$ is less than $1 / 4$ (while $f_{1}$ vanishes). Therefore, the component $f_{2}$ for any $T_{a, \delta}$ must be less than $1 / 4$ and greater than 0 (because the zero value is reached for the limit tori $T_{0, \delta} \subset Q_{\mu}$, which are degenerate). But this is impossible for a Bohr-Sommerfeld Lagrangian torus of level 1, 2, or 3.

Hence, although the last example is excluded from our main case of real polarizations with regular degenerations, we can use arguments related to the the action map that arises naturally in the regular case. Below, we discuss this question in more detail.

We consider an arbitrary polarization $\pi: \mathbb{C P}^{2} \rightarrow B \subset \mathbb{R}^{2}$, where $B$ is a convex polytope. We assume that it has only regular degenerations. This means that there exists a set of symplectic divisors $D_{1}, \ldots, D_{m} \subset \mathbb{C P}^{2}$ such that $\operatorname{dim}_{\mathbb{R}} D_{i}=2$. These divisors are projected to the edges of $B$. It then follows that each $D_{i}$ represents the class $[D] \in H_{2}\left(\mathbb{C P}^{2}, \mathbb{Z}\right)$ Poincaré dual to the cohomology class $[\omega]$ and that the number of symplectic divisors is $\operatorname{deg} K^{-1}=3$.

Indeed, the total degree of the degeneration components must be the degree of the anticanonical class because the "inner" part of $\mathbb{C P}^{2}$ with respect to $B$ admits a nonvanishing holomorphic vector bifield of the type $(2,0)$ with respect to an arbitrary almost complex structure compatible with $\omega$ and $\pi$. Each component in $\pi^{-1}(\partial B)$ must have a positive degree with respect to $[\omega] \in H^{2}\left(\mathbb{C P}^{2}, \mathbb{Z}\right)$. On the other hand, the number of components is equal to the number of edges of our convex polytope $B$, i.e., it must be greater than or equal to three. It is hence clear that for $\mathbb{C P}^{2}$, the regularity dictates the form of $B$ and the type of $\pi^{-1}(\partial B)$.

Furthermore, the analysis of the system becomes simpler if we can find integrals of a special type. Because the "inner" part of $\mathbb{C P}^{2}$ is topologically equivalent to the direct product $(B-\partial B) \times T^{2}$, we can choose a basis in $H_{1}\left(\pi^{-1}(b), \mathbb{Z}\right)$ uniformly for all smooth fibers of $\pi$. Moreover, we can do this with respect to the boundary components $D_{1}, D_{2}$, and $D_{3}$ if we choose any two of the three. The point is that for any $D_{i}$, there exists a uniquely determined basis element in $H_{1}\left(\pi^{-1}(b), \mathbb{Z}\right)$ that degenerates in the passage to a limit fiber in $D_{i}$. This means that we have distinguished primitive elements $d_{1}, d_{2}, d_{3} \in H_{1}\left(\pi^{-1}(b), \mathbb{Z}\right)$ such that each pair of $d_{i}$ and $d_{j}, i \neq j$, form a basis. We choose and fix $d_{1}$ and $d_{2}$ as a basis. Then there exists a lift of the period map with respect to the boundary data

$$
p_{d_{1}, d_{2}}=\left(p_{d_{1}, d_{2}}^{1}, p_{d_{1}, d_{2}}^{2}\right): B \rightarrow \mathbb{R}^{2}
$$

such that $p_{d_{1}, d_{2}}$ is smooth on $B-\partial B,\left.p_{d_{1}, d_{2}}^{i}\right|_{\pi\left(D_{i}\right)}=0$, and $p_{d_{1}, d_{2}}^{i}(b)=\int_{D} \omega(\bmod \mathbb{Z})$, where $D \subset \mathbb{C P}^{2}$ is a disc with the boundary $\partial D=\gamma_{d_{i}} \subset \pi^{-1}(b)$ and $\left[\gamma_{d_{i}}\right]=d_{i} \in H_{1}\left(\pi^{-1}(b), \mathbb{Z}\right)$. Such a lift exists, and there are exactly four possibilities for the extension $p_{d_{1}, d_{2}}$ because there are exactly four possible choices of the signs of $d_{1}$ and $d_{2}$ (cf. the discussion on the choice of spin structures in [3], [11]). We fix the signs such that $p_{d_{1}, d_{2}}$ is nonnegative on $B$.

We note that such a lift gives us a certain set of real functions that are natural generalizations of the classical action variables for an integrable mechanical system. It is therefore natural to call it the action map. 
Let $a_{i j}=D_{i} \cap D_{j}$. Then it is easy to see that

$$
p_{d_{1}, d_{2}}\left(a_{12}\right)=(0,0), \quad p_{d_{1}, d_{2}}\left(a_{13}\right)=(0,1), \quad p_{d_{1}, d_{2}}\left(a_{23}\right)=(1,0) .
$$

It hence follows that

$$
d_{3}=d_{1}+d_{2} \in H_{1}\left(\pi^{-1}(b), \mathbb{Z}\right) .
$$

Indeed, $d_{3}$, being a primitive element, can be represented as $d_{3}=p d_{1}+q d_{2}$, where $p$ and $q$ are coprime integers. But the symplectic area is an additive functional, and it is hence clear that $p=q=1$.

We now use the fact, proved in Sec. 2, that the Kodaira-Spencer map is an isomorphism. This implies one very important property of our action map.

Lemma. The function $p_{d_{1}, d_{2}}$ is strictly monotonic in both arguments.

Indeed, because each component of $p_{d_{1}, d_{2}}$ is monotonic on the corresponding boundary and because the Kodaira-Spencer map in this case coincides with the differential of $p_{d_{1}, d_{2}}$, we see that the lifted period map $p_{d_{1}, d_{2}}$ does not have any critical points on $B-\partial B$ and the restriction $\left.p_{d_{1}, d_{2}}^{j}\right|_{L_{c}}$ is a strictly monotonic (increasing) function for any level line $L_{c}=\left\{p_{d_{1}, d_{2}}^{i}=c, 0 \leq c<1\right\}$.

We now verify Conjectures $2-4$ in this case.

It follows from the monotonicity of $p_{d_{1}, d_{2}}$ that

$$
0<p_{d_{1}, d_{2}}^{1}(b)+p_{d_{1}, d_{2}}^{2}(b)<1
$$

for any regular fiber $S_{b}=\pi^{-1}(b), b \in B-\partial B$. By the definitions of $p_{d_{1}, d_{2}}$ and of a Bohr-Sommerfeld fiber of level $k$, we find that the minimal possible nonempty level is level 3 , and Conjecture 2 holds.

From the monotonicity of $p_{d_{1}, d_{2}}$, we find that there exists only one Bohr-Sommerfeld fiber of level 3 with respect to the canonical class. We note that for this fiber $S_{\text {can }}$, we have

$$
p_{d_{1}, d_{2}}^{1}\left(S_{\mathrm{can}}\right)=p_{d_{1}, d_{2}}^{2}\left(S_{\mathrm{can}}\right)=\frac{1}{3}
$$

To prove the monotonicity of $S_{\text {can }}$, it suffices to find for each generator of $H_{1}\left(S_{\text {can }}, \mathbb{Z}\right)$, a smooth loop $\gamma$ representing this generator and a smooth disc $D$ bounded by $\gamma$ such that the Maslov index of $[\gamma, D]$ would be three times the symplectic area of $D$. This suffices because the relation is the same for any other disc $D^{\prime}$ with the same boundary $\gamma$ because $\mathbb{C P}^{2}$ is monotonic. We note that because the set of Lagrangian fibers is connected the Maslov index is the same for all Lagrangian tori.

For our distinguished generator $d_{1} \in H_{1}\left(S_{\text {can }}, \mathbb{Z}\right)$, we choose a smooth loop $\gamma_{1} \subset S_{\text {can }}$ such that $\left[\gamma_{1}\right]=d_{1}$. We consider the level line $C_{1 / 3}=\left\{p_{d_{1}, d_{2}}^{2}=1 / 3\right\}$ and choose the segment $B_{t} \subset C_{1 / 3}, t \in[0,1 / 3]$, which corresponds to the inequality $p_{d_{1}, d_{2}}^{1} \leq 1 / 3$. There exists a family of smooth loops $\left\{\gamma_{1}^{t}\right\}, t \in[0,1 / 3]$, such that

$$
\gamma_{1}^{1 / 3}=\gamma_{1} \subset S_{\text {can }}, \quad \pi\left(\gamma_{1}^{t}\right)=b(t) \in B_{t} \subset B, \quad \gamma_{1}^{t} \subset S_{b(t)}, \quad\left[\gamma_{1}^{t}\right]=d_{1} \in H_{1}\left(S_{b(t)}\right) .
$$

This family $\left\{\gamma_{1}^{t}\right\}$ shrinks to the point $\gamma_{1}^{0}$ on the symplectic divisor $D_{1}$. It is easy to see that the family $\left\{\gamma_{1}^{t}\right\}$ forms a disc

$$
\bigcup_{t \in[0,1 / 3]} \gamma_{1}^{t}=D \subset \mathbb{C P}^{2}
$$

and hence

$$
\int_{D} \omega=\frac{1}{3} .
$$


On the other hand, the Maslov index of $\left[\gamma_{1}, D\right]$ is equal to 1 . Indeed, because we shrink $\gamma_{1}$ to a point over the level line of $p_{d_{1}, d_{2}}^{2}$, it follows that the Maslov index of $D$ must be the degree of the normal bundle of $D_{1}$. We hence have

$$
\mu\left(\left[\gamma_{1}, D\right]\right)=1=3 \cdot \frac{1}{3}=3 \int_{D} \omega
$$

and because we can repeat the arguments for a smooth loop $\gamma_{2} \subset S_{\text {can }}$ representing the generator $d_{2}$, it follows that $S_{\text {can }}$ is monotonic. Conjecture 3 holds.

We prove that if a fiber $S_{b}$ is not Bohr-Sommerfeld with respect to the canonical class, then it is displaceable. For this, it suffices to prove that the same happens for a fiber that is not on the "diagonal" $\left\{p_{d_{1}, d_{2}}^{1}=p_{d_{1}, d_{2}}^{2}\right\}$. Indeed, our choice of the pair $\left(d_{1}, d_{2}\right)$ was arbitrary, and taking another pair, for example, $\left(d_{1}, d_{3}\right)$, we obtain the same result for the corresponding "diagonal," and because the intersection of the "diagonals" is exactly one point, which is Bohr-Sommerfeld with respect to the canonical class, it follows that Conjecture 4 holds for fibers of a real polarization of $\mathbb{C P}^{2}$ with regular degenerations.

We claim that there exists a Hamiltonian deformation of $\mathbb{C P}^{2}$ that generates the corresponding Hamiltonian isotopy interchanging fibers with the values $\left(c_{1}, c_{2}\right)$ and $\left(c_{2}, c_{1}\right)$ with respect to the function $p_{d_{1}, d_{2}}^{i}$. The required Hamiltonian deformation is constructed explicitly as follows. We consider the level lines of the sum $p_{d_{1}, d_{2}}^{1}+p_{d_{1}, d_{2}}^{2}$ lifted to $\mathbb{C P}^{2}$. The possible values are in $[0,1]$. There are two exceptional level sets: we have the point $D_{1} \cap D_{2}$ for $c=0$ and $D_{3}$ for $c=1$. For any other $\alpha \in(0,1)$, the level line

$$
C_{\alpha}=\pi^{-1}\left(\left\{p_{d_{1}, d_{2}}^{1}+p_{d_{1}, d_{2}}^{2}=\alpha\right\}\right)
$$

is a smooth three-dimensional sphere. The restriction of the symplectic form $\omega$ to $C_{\alpha}$ defines a fibration

$$
p_{\alpha}: C_{\alpha} \rightarrow S_{\alpha}^{2}
$$

topologically isomorphic to the Hopf bundle. Indeed, we take the kernels of $\left.\omega\right|_{C_{\alpha}}$, and the corresponding one-dimensional distribution is integrable, which gives the fibration. In addition, we have a symplectic form $\omega_{\alpha}$ on $S_{\alpha}^{2}$ that is the result of the reduction applied to $\omega$ and a smooth circle $S_{\alpha}^{1} \subset S_{\alpha}^{2}$ that is the result of the phase factorization of the "diagonal" torus with the periods $(\alpha / 2, \alpha / 2)$.

We note that as $\alpha \rightarrow 1$, this Hopf bundle $C_{\alpha} \rightarrow S_{\alpha}^{2}$ degenerates to $D_{3}$ with a marked circle $S_{1}^{1} \subset D_{3}$. Moreover, the triple $\left(D_{3},\left.\omega\right|_{D_{3}}, S_{1}^{1}\right)$ is the result of the limit transition for $\left(S_{\alpha}^{2}, \omega_{\alpha}, S_{\alpha}^{1}\right)$ as $\alpha \rightarrow 1$. On the other hand, the opposite limit as $\alpha \rightarrow 0$ is realized as a conformal shrinking of the triple $\left(S_{\alpha}^{2}, \omega_{\alpha}, S_{\alpha}^{1}\right)$ to the point $D_{1} \cap D_{2}$. Indeed, it is clear that the symplectic volume is

$$
\int_{S_{\alpha}^{2}} \omega_{\alpha}=\alpha
$$

On the symplectic two-dimensional sphere $D_{3}$, we choose a smooth function $f_{1} \in C^{\infty}\left(D_{3}, \mathbb{R}\right)$ such that $f_{1}$ is a height function and both its two nondegenerate critical points $p_{1}^{\mathrm{N}}$ and $p_{1}^{\mathrm{S}}$ are on the marked circle $S_{1}^{1}$. Using the inverse limit process, we can now construct a family of smooth functions $\left\{f_{\alpha}\right\}$ for the family of two-dimensional spheres $\left\{S_{\alpha}^{2}\right\}$ for $\alpha \in(0,1]$. We take an appropriate normalization for the functions,

$$
\int_{S_{\alpha}^{2}} f_{\alpha} \omega_{\alpha}=\alpha^{2}
$$

and then lift each function $f_{\alpha}$ to $C_{\alpha}$ via the canonical projection

$$
F_{\alpha}=f_{\alpha} \circ p_{\alpha}: C_{\alpha} \rightarrow \mathbb{R}
$$


We claim that these lifted functions can be combined into a smooth global function $F$ such that

$$
\left.F\right|_{C_{\alpha}}=F_{\alpha}
$$

This function has exactly three critical points: the intersection point $D_{1} \cap D_{2}$ and the two points $p_{1}^{\mathrm{N}}$ and $p_{1}^{\mathrm{S}}$ on $D_{3}$.

The flow on $\mathbb{C P}^{2}$ generated by the Hamiltonian vector field $X_{F}$ is a one-parameter family of symplectomorphisms $\phi_{t}$ of $\mathbb{C P}^{2}$ such that $\phi_{2 \pi}=$ id. This family corresponds to the rotation of the sphere $S_{\alpha}^{2}$ about the fixed points $p_{\alpha}^{\mathrm{N}}$ and $p_{\alpha}^{\mathrm{S}}$ on the "diagonal" circle $S_{\alpha}^{1}$. It is easy to see that the result of a certain rotation applied to a fiber of the given real polarization with the periods $\left(c_{1}, c_{2}\right)$ is the fiber with the periods $\left(c_{2}, c_{1}\right)$. This completes the proof of Conjecture 4 for fibers of a real polarization with regular degenerations.

Summarizing the discussion, we obtain the following theorem.

Theorem 3. Conjecture 1 holds for $\mathbb{C P}^{2}$.

We note that the method of the lifted period function, i.e., the method of the action map, can be applied to any compact simply connected symplectic manifold, and the main property of the Kodaira-Spencer map can be used to establish the strict monotonicity of the lifted period function, which was crucial in our construction for $\mathbb{C P}^{2}$ above. We can therefore expect that our method will also be useful in more general cases, for other monotonic symplectic manifolds.

At the same time, before studying Conjectures 2-4, which were formulated for any Lagrangian tori in $\mathbb{C P}^{2}$, we could try to find the answer to the following natural question. Is there a geometric condition on a Lagrangian torus in $\mathbb{C P}^{2}$ that detects whether this torus can be included in a family of Lagrangian fibers of a real polarization with regular degenerations?

Acknowledgments. The author thanks the Max-Planck-Institut (Bonn) for the hospitality, the excellent working conditions, and the opportunity to communicate with high-level mathematicians. He is grateful to F. Hirzebruch, A. Gorodentsev, P. Pushkar, A. Kokotov, P. Moree, and many others. The author must thank D. Auroux and D. Orlov for the constant help and useful remarks. Last but not least, the author thanks the staff of the Max-Planck-Institut.

This work was supported in part by the Russian Foundation for Basic Research (Grant Nos. 08-0100095, 08-01-00392, and 07-01-92211) and the Program for Supporting Leading Scientific Schools (Grant No. NSh-1987.2008.1).

\section{REFERENCES}

1. M. Kontsevich, "Homological algebra of mirror symmetry," in: Proc. Intl. Congress Math., Zürich (S. D. Chatterji, ed.), Vols. 1 and 2, Birkhäuser, Basel (1995), pp. 120-139.

2. K. Hori and C. Vafa, "Mirror symmetry," arXiv:hep-th/0002222v3 (2000).

3. C.-H. Cho and Y.-G. Oh, Asian J. Math., 10, 773-814 (2006).

4. V. Guillemin and S. Sternberg, Symplectic Technique in Physics, Cambridge Univ. Press, Cambridge (1990).

5. J. Śniatycki, Geometric Quantization and Quantum Mechanics (Appl. Math. Sci., Vol. 30), Springer, New York (1980).

6. N. Woodhouse, Geometric Quantization, Oxford Univ. Press, Oxford (1980).

7. N. Tyurin, "Geometric quantization and algebraic Lagrangian geometry," in: Surveys in Geometry and Number Theory: Reports on Contemporary Russian Mathematics (London Math. Soc. Lecture Note Ser., Vol. 338, N. Young, ed.), Cambridge Univ. Press, Cambridge (2007), pp. 279-318.

8. A. Tyurin, "Geometric quantization and mirror symmetry," arXiv:math/9902027v1 (1999).

9. A. Weinstein, Ann. of Math., 98, 377-410 (1973). 
10. N. A. Tyurin, Theor. Math. Phys., 150, 278-287 (2007).

11. C.-H. Cho, "Holomorphic discs, spin structures, and the Floer cohomology of the Clifford torus," Doctoral dissertation, Univ. of Wisconsin, Madison (2003); Int. Math. Res. Not., 2004, No. 35, 1803-1843 (2004).

12. Yu. Chekanov and T. Schlenk, "Lagrangian tori in projective spaces," (in preparation).

13. D. Auroux, J. Gőkova Geom. Topol., 1, 51-91 (2007); arXiv:0706.3207v2 (2007).

14. S. Belev, "Proper nonlinear quantum subsystems of standard quantum systems [in Russian]," Baccalureate thesis, Joint Inst. Nucl. Res., Dubna (2007). 